\title{
Influencing Factors on Post-Dialysis Fatigue in Hemodialysis Patients
}

\author{
Su-Jeong Han ${ }^{1}$ and Hye-Won Kim² \\ 1, first author Department of Nursing, Konyang University, \\ sjhan@konyang.ac.kr \\ 2, corresponding author Seoul Women's College of Nursing, \\ hwkim@snjc.ac.kr
}

\begin{abstract}
The purpose of this study was to measure post-dialysis fatigue in patients on hemodialysis and identifies clinical variables that are associated with post-dialysis fatigue. The subjects for this study were 103 patients who were receiving chronic hemodialysis in outpatient dialysis clinics in Seoul between April $12^{\text {th }}$ and April $23^{\text {th }}, 2010$ and consented to participate in the study. The collected data were analyzed by the SPSS WIN 12.0 program. The mean score of fatigue were 63.4, 86.8 and 60.8 before hemodialysis, after hemodialysis and next day, respectively. There was a statistically significant ( $F=5.16, \mathrm{p}<.001)$. All these indicators but 'tired', 'not energetic', 'difficult to move', 'would like to lie' did not significantly differ over time after hemodilaysis. 'Would like to lie' was strongest after hemodialysis $(F=12.52, p<.001)$. There were significantly positive correlations between post-dialysis fatigue and weight gain ( $r=.18, p=.038$ ). There were significantly positive correlations between weight gain and 'tired', 'difficult to move', and 'would like to lie'. There were also significantly positive correlations between post-dialysis fatigue and age $(r=.19, p=.035)$, comorbidities $(r=.14, p=.031)$, post dialysis blood pressure $(r=-.17, p=.024)$, weight change $(r=.14, p=.034)$, and hemoglobin ( $r=-.27, p=.005)$. Also, Age, comorbidities post-dialysis blood pressure, weight change, and hemoglobin predicted value accounted for $16.8 \%$ of the variance on post-dialysis fatigue $(F=13.08, p<.001)$. It is considered that dialysis providers care to recognize post-dialysis fatigue as an important nursing issue for hemodialysis patients. It is also necessary to develop a nursing intervention for improving their post-dialysis fatigue.
\end{abstract}

Keywords: hemodialysis, post-dialysis fatigue, nursing

\section{Introduction}

Fatigue is one of the most frequent complaints of dialysis patients and is associated with impaired health-related quality of life (HRQOL). The prevalence of fatigue ranges from $60 \%$ to as high as $97 \%$ in patients on long-term renal replacement therapy. The importance of fatigue to patients with kidney disease is underscored by the observation that $94 \%$ of hemodialysis patients endorsed a willingness to undergo more frequent dialysis if there would be an associated increase in energy level $[1,2]$. Post- hemodialysis fatigue is a common, often incapacitating symptom and may be improved with more frequent treatment. Lindsay, et al., looked at post-dialysis fatigue in 45 subjects and found a positive association between "time to recover (minutes) from HD" and fatigue; patients with longer recovery time tended to have greater levels of fatigue [3]. Also, the relationship between recovery time and fatigue was strongest immediately after 
dialysis and weakened progressively during the time between sessions. In this study, the time to recover from HD also showed a significant positive association with the total dialysis stress score, which encompasses an array of physical signs and symptoms that can arise during HD procedure [4]. Ultrafiltration, diffusion, osmotic disequilibrium, changes in blood pressure, blood membrane interactions, higher levels of tumor necrosis factor, and psychological factors like depression have all been implicated in the pathogenesis of post-dialysis fatigue [5]. A number of physiological disorders are associated with fatigue, including anemia and other chronic illnesses such as rheumatoid arthritis, infection, and renal disease. Anemia reduces the oxygen supply to the tissues and is frequently cited as one of the main contributing factors to fatigue in renal failure, resulting from reduced erythropoietin production. Fatigue may indicate to the individual that illness is present and motivate them to seek treatment. The purpose of this study was to measure postdialysis fatigue in patients on hemodialysis and identifies clinical variables that are associated with post-dialysis fatigue.

\section{Method}

\subsection{Subjects and Data Collection}

The subjects for this study were 103 patients who were receiving chronic hemodialysis in outpatients' dialysis clinic in Seoul between April $12^{\text {th }}$ and April $23^{\text {th }}, 2010$ and consented to participate in the study. Those who agreed to answer and participate were given the questionnaire. Data were collected using face-to-face interview with a structured questionnaire. It took 10 minutes to complete the questionnaire. An Electronic Medical Record (EMR) was referred to regarding comorbidities, hemoglobin, post-dialysis blood pressure, pre-dialysis blood pressure, and weight change.

\subsection{Instruments}

2.2.1. Post-dialysis Fatigue: The assessment of post-dialysis fatigue of subjects was first invented by Lee, Hicks and Nino-Murcia (1991) and them modified by Kim (1995) [6]. The total score ranges from 0-100mm Visual Analogue Scale for each subscale. The total indicators of fatigue level were 16: 'tired', 'sleepy', 'dull', 'languid', 'not energetic', 'powerful', 'vigorous', 'efficient', 'vital', 'exhausted', 'burnout', 'difficult to open eyes', 'difficult to move',' difficult to concentrate', 'difficult to talk' and 'would like to lie'.

2.2.2. Other Variables: Gender, age, educational level, comorbidities, hemoglobin, post-dialysis blood pressure, pre-dialysis blood pressure, and weight change (interdialytic weight gain) were determined from interviews and EMR reviews.

\subsection{Data Analysis}

Collected data were statistically analyzed with the SPSS WIN (ver. 12.0) program. Analysis included percentage, average, standard deviation, Pearson's correlation coefficient, ANOVA, and multiple regressions.

\subsection{Ethical Consideration}

Standard ethical and legal points were followed regarding the use of reporting subjects in research; salient, relative points were explained to all subjects. These guidelines included: participants right to withdraw from the project, anonymity, 
limitations on the use of resulting data, use for research and or academic purposes only, and the possible destruction of sensitive materials.

\section{Results}

\subsection{General Characteristics of the Patients}

The general characteristics are presented in Table 1. Fifty-four point four percent $(54.4 \%)$ of patients were male, with a mean age of 61.2 years.

Table 1. General Characteristics of Patients

\begin{tabular}{lcc} 
& $\mathrm{N}=103$ \\
\hline Variable & $\mathrm{N}(\%)$ or Mean $\pm \mathrm{SD}$ \\
\hline Gender & $\begin{array}{c}\text { Male } \\
\text { Female }\end{array}$ & $56(54.4)$ \\
Age & $47(45.6)$ \\
Years of Education & $61.2 \pm 11.3$ \\
Comorbidities & $10.2 \pm 3.3$ \\
Post-dialysis blood pressure $(\mathrm{mm} / \mathrm{Hg})$ & $2.5 \pm 0.9$ \\
Pre-dialysis blood pressure $(\mathrm{mm} / \mathrm{Hg})$ & $123 \pm 20 / 76 \pm 13$ \\
Weight change $(\mathrm{kg})$ & $146 \pm 19 / 90 \pm 16$ \\
Hemoglobin(g/dl) & $2.1 \pm 0.7$ \\
\hline
\end{tabular}

\subsection{Post-dialysis Fatigue of Hemodialysis Patients}

The total level of fatigue scored on average 63.4, 86.8 and 60.8 before hemodialysis, after hemodialysis, and next day, respectively <Table $2>$. There was a statistically significant $(\mathrm{F}=5.16, p<.001)$. All these indicators but 'tired', 'not energetic', 'difficult to move', 'would like to lie' did not significantly differ over time after hemodialysis. 'Would like to lie' was strongest after hemodialysis ( $\mathrm{F}=12.52$, $p<.001)$. 
Table 2. Post Dialysis Fatigue of Hemodialysis Patients

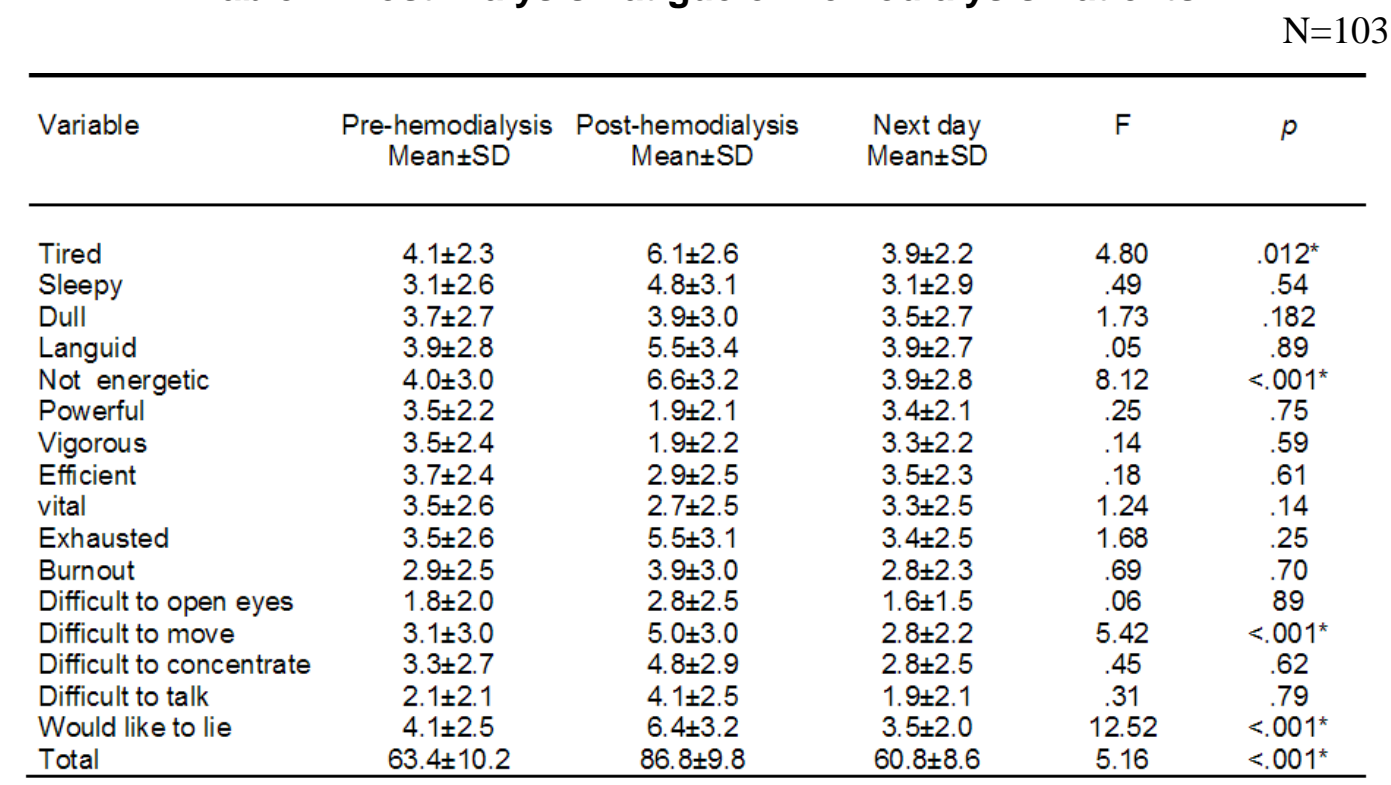

$* p<.05$

\subsection{Correlation between Post-dialysis Fatigue and Weight Gain}

There were significantly positive correlations between post-dialysis fatigue and weight gain $(\mathrm{r}=.18, p=.038)<$ Table 3 >. There were also significantly positive correlations between weight gain and 'tired', 'difficult to move', and 'would like to lie'.

Table 3. Correlation between Post-dialysis Fatigue and Weight Gain

\begin{tabular}{lcc}
\hline & Weight gain & $p$ \\
& $r$ & \\
\hline & & $.038^{*}$ \\
Post-dialysis fatigue & .18 & $.042^{*}$ \\
Tired & & .154 \\
Sleepy & .13 & .424 \\
Dull & .11 & .416 \\
Languid & .07 & .154 \\
Not energetic & .06 & .067 \\
Powerful & -.11 & .134 \\
Vigorous & -.13 & .182 \\
Efficient & -.11 & .382 \\
Vital & -.10 & .542 \\
Exhausted & -.07 & .482 \\
Burnout & .04 & .438 \\
Difficult to open eyes & .06 & $.041^{*}$ \\
Difficult to move & .07 & .201 \\
Difficult to concentrate & .18 & .386 \\
Difficult to talk & .09 & $.002^{*}$ \\
Would like to lie & .07 & .29 \\
\hline
\end{tabular}

$$
* p<.05
$$




\subsection{Correlation between Post-dialysis Fatigue and other Variables}

There were significantly positive correlations between post-dialysis fatigue and age $(\mathrm{r}=.19, p=.035)$, comorbidities $(\mathrm{r}=.14, p=.031)$, post dialysis blood pressure $(\mathrm{r}=-$ $.17, p=.024)$, weight change $(\mathrm{r}=.14, p=.034)$ and hemoglobin $(\mathrm{r}=-.27, p=.005)$ $<$ Table 4>.

Table 4. Correlation between post-dialysis fatigue and other variables

\begin{tabular}{lcc}
\hline & Post-dialysis fatigue & $p$ \\
& $\mathrm{r}$ & \\
\hline Gender & .03 & .621 \\
Age & .19 & $.035^{*}$ \\
Years of Education & -.02 & .692 \\
Comorbidities & .14 & $.031^{*}$ \\
Post-dialysis blood pressure $(\mathrm{mm} / \mathrm{Hg})$ & -.17 & $.024^{*}$ \\
Pre-dialysis blood pressure $(\mathrm{mm} / \mathrm{Hg})$ & .05 & .452 \\
& .14 & $.034^{*}$ \\
Weight change $(\mathrm{kg})$ & -.27 & $.005^{*}$ \\
Hemoglobin $(\mathrm{g} / \mathrm{dl})$ & & \\
& & \\
\hline
\end{tabular}

$$
* p<.05
$$

\subsection{Factors of Affecting the Post-dialysis Fatigue in Hemodialysis Patients}

Age $(p=.002)$, comorbidities $(p=.013)$, post-dialysis blood pressure, weight change and hemoglobin $(p<.001)$ predicted value accounted for $16.8 \%$ of the variance on post-dialysis fatigue $(\mathrm{F}=13.08, p<.001)<$ Table $5>$.

Table 5. Factors of Affecting the Post-dialysis Fatigue in Patients

\begin{tabular}{|c|c|c|c|c|}
\hline Factor variables & $\begin{array}{c}\text { Standardized } \\
\text { Beta }\end{array}$ & $\mathrm{t}$ & $p$ & Adj $R^{2}$ \\
\hline Constant & & & & .168 \\
\hline Age & .095 & 1.18 & .239 & \\
\hline $\begin{array}{l}\text { Comorbidities } \\
\text { Post-dialysis blood pressure }(\mathrm{mm} / \mathrm{Hg}) \\
\text { Weight change }(\mathrm{kg}) \\
\text { Hemoglobin }(\mathrm{g} / \mathrm{dl})\end{array}$ & $\begin{array}{l}.146 \\
.502 \\
.311 \\
.461\end{array}$ & $\begin{array}{l}.185 \\
6.53 \\
3.37 \\
4.98\end{array}$ & $\begin{array}{l}.064 \\
<.001^{*} \\
.012^{*} \\
<.001^{*}\end{array}$ & \\
\hline
\end{tabular}

\section{Discussion}

This study was to measure the post-dialysis fatigue in patients on hemodialysis and to identify clinical variables that are associated with post-dialysis fatigue. We found that in hemodialysis, patients with CKD had high levels of post-dialysis fatigue. Also, clinical variables such as age, comorbidities, post-dialysis blood 
pressure, weight change, and hemoglobin level were identified to have a significant relationship. The prevalence of post-dialysis fatigue was identified in $59.8 \%$ of the patients. This finding was similar to that of several other studies. The patients report that they feel worse in the immediate hours after the dialysis session, while one out of four reports severe or very severe intensity of fatigue after dialysis [7]. The severity of "Post-dialysis Fatigue" symptoms could range from mild to severe and can last from a few hours after the dialysis procedure up to until the next day $[4,8]$ or for a "very long time" [7]. Thus, many HD patients may spend a large proportion of their time in a state of fatigue [7, 9]. Since they perceive fatigue (whether in dialysis or in non-dialysis days) as an important barrier [10], this adversely affects their physical activity levels. Regarding study results, post-dialysis fatigue was associated with post-dialysis blood pressure, weight change, and hemoglobin. There are several possible explanations for this finding. First, anemia reduces the oxygen supply to the tissues and is frequently cited as one of the main contributing factors to fatigue in renal failure, resulting from reduced erythropoietin production. It is suggested that more attention needs to be centered on the symptomatic effects of anemia. Second, inter-dialytic weight gain is mainly the result of salt and water intake between two dialysis sessions. Theoretically, the consequences of this variable have a double meaning. On the one hand, the water and saline intake can frequently be done together with caloric and protein foods, which mean it would be associated with a better nutritional status [11]. But, on the other hand, water and salt intake can give rise to a volume overload, which can be the key for the development of high blood pressure and left ventricle hypertrophy, both of which can increase cardiovascular risk [12]. Third, hypertension may be present in up to $80 \%$ of patients reaching end-stage renal disease [13], sometimes as a primary cause of renal failure but more usually as a secondary complication. In dialysis patients the commonly attributed to increased circulating intravascular volume, so-called volume-dependent hypertension, which is partially assessed by the inter-dialytic weight gain and controlled by fluid removal during dialysis to the appropriate dry weight. This practice assumes a direct relationship between weight gain and increased blood pressure, and indeed inter-dialytic weight gain has been directly related to cardiovascular mortality in hemodialysis patients [14]. Hypotension is also a major clinical problem not only because of its frequency, but also because it contributes to the un-well feelings experienced by dialysis patients, limits rehabilitation, and consumes a disproportionate amount of dialysis staff time and resources. Dialysis hypotension has a multifactorial etiology, including such disparate causes as autonomic, dysfunction, decreased plasma osmolality, and a decrease in extracellular fluid volume with inadequate plasma [15]. The importance of fatigue in patients with kidney disease was emphasized by the observation that 94\% of the patients who were undergoing hemodialysis endorsed a willingness to undergo more frequent dialysis if there was an associated increase in their energy level [16]. Fatigue influences individuals' role performance and functional status [17]. Despite the importance of fatigue in patients, healthcare providers remain largely unaware of the presence and severity of fatigue in patients who undergo dialysis. Education programs for renal nurses should emphasize the importance of a holistic approach to care rather than overemphasizing technological aspects. Specific knowledge and skills to undertake assessment and provide care to deal with the rage of post-dialysis fatigue symptoms must be included. Such changes have the potential to enhance the quality of life for these patients. As a conclusion of these findings, it is considered that health care providers should care to recognize postdialysis fatigue as an important nursing issue for hemodialysis patients. It is also necessary to assess the impact of frequent and novel dialysis techniques on postdialysis fatigue. 


\section{References}

[1] S. D. Weisbord, L. F. Fried, R. M. Arnold, et al., J Am Soc Nephrol., vol. 16, no. 2487, (2005).

[2] N. Ramkumar, S. Beddhu, P. Eggers, L. M. Pappas and A. K. Cheung, Hemodial Int., vol. 9, no. 281, (2005).

[3] B. O. Lee, C. C. Lin, W. Chaboyer, C. L. Chiang and C. C. Hung, J Clin Nur., vol. 16, no. 407, (2007).

[4] R. M. Lindsay, P. A. Heidenheim, G. Nesrallah, A. X. Garg and R. Suri, Clin J Am Soc Nephrol., vol. 1, no. 952 , (2006).

[5] A. H. Sklar, L. A. Riesenberg, A. K. Silber, W. Ahmed and A. Ali, Am J Kidney Dis., vol. 28, no. 732, (1996).

[6] H. R. Kim, J Kor Academy Nur., vol. 26, no. 53, (1996).

[7] P. L. Gordon, J. W. Doyle and K. L. Johansen, Clin. Nephrol., vol. 75, no. 426, (2011).

[8] S. J. Han and H. W. Kim, "Healthcare and Nursing", Proceedings of the 7th International Congress of Advanced Science and Technology Letters, (2015) August 19-21, Jeju island, Korea.

[9] B. Caplin, S. Kumar and A. Davenport, Nephrol. Dial. Transplant, vol. 26, no. 2656, (2011).

[10] C. Delgado and K. L. Johansen, Nephrol. Dial. Transplant, vol. 27, no. 1152, (2012).

[11] S. Sezer, F. N. Ozdemir, Z. Arat, et al., Renal Fail, vol. 24, no. 37, (2002).

[12] M. Rahman, P. Fu, A. R. Sehgal and M. C. Smith, Am J Kidney Dis., vol. 35, no. 257, (2000).

[13] J. Herrera-Acosta, Kidney Int., vol. 19, no. 505, (1992).

[14] P. Kramer, M. Broyer, F. B. Brunner, et al., Proc EDTA-ERA, vol. 21, no. 2, (1985).

[15] D. Sunita and L. H. William, Kidney Int., vol. 59, no. 1175, (2001).

[16] M. Jhamb, S. D. Weisbord, J. L. Steel and M. Unruh, Am J Kidney Dis., vol. 52, no. 353, (2008).

[17] K. McCann and R. P. Boore, J Adv Nur., vol. 32, no. 1132, (2000).

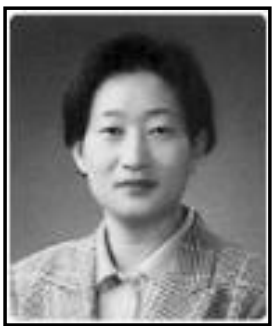

\section{Su-Jeong Han}

Feb. 2001: Ewha Womans Univ. PhD.

Feb. 1997 - Current: Konyang Univ. Professor

Research Interests: Medical-surgical nursing, chronic disease care

E-Mail: sjhan@konyang.ac.kr

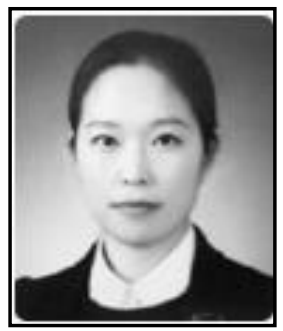

\section{Hye-Won Kim}

Feb. 2012: Seoul National Univ. PhD.

Aug. 2014 - Current: Seoul Women's College of Nursing, Professor

Research Interests: Chronic Kidney Disease, Biobehavioral factors E-Mail: hwkim@snjc.ac.kr 
International Journal of $u-$ and e- Service, Science and Technology Vol.8, No. 10 (2015) 